\title{
IFN- $\gamma$ induction by neutrophil-derived IL-17A homodimer augments pulmonary antibacterial defense
}

\author{
S Cai ${ }^{1}$, S Batra ${ }^{1,4}$, I Langohr ${ }^{1}$, Y Iwakura ${ }^{2}$ and S Jeyaseelan ${ }^{1,3}$
}

The role of interleukin-17A (IL-17A) in host defense against Legionella pneumophila remains elusive. To address this issue, we used $/ 117 a^{-1-}, \| 17 f^{-\prime-}$, and $/ 117 a / I 17 f^{-\prime-}$ mice on a C57BI/6 (non-permissive) background and IL-17 neutralizing Abs in mice on an $\mathrm{A} / \mathrm{J}$ (permissive) background. Higher bacterial (L. pneumophila) counts in the lung and blood along with reduced neutrophil recruitment were detected in $I 17 a^{-I_{-}^{-}}$, but not $I 17 f^{-I_{-}}$, mice. We found that neutrophils produce IL-17A homodimer (IL-17A) during L. pneumophila infection, and hematopoietic cell-derived IL-17A is known to be important for bacterial clearance. Thus, intratracheal administration of wild-type neutrophils or recombinant IL-17A restored bacterial clearance and neutrophil recruitment in $/ 17 \mathrm{a}^{-1-}$ mice. Furthermore, neutrophil-depleted Rag2 $^{-1-}$ and Rag2/II-2 $\mathrm{r \gamma}^{-/-}$mice exhibited increased bacterial burden, reduced neutrophil influx and IL-17A production in the lung. Recombinant IFN- $\gamma$ administration in $/ 17 a^{-1-}$ mice augmented bacterial elimination, whereas IL-17A administration in Ifn $\gamma^{-1-}$ mice did not augment bacterial clearance. IFN- $\gamma$ is produced by $\mathrm{T}$ cells, but not neutrophils or macrophages, suggesting that neutrophil-derived IL-17A induces IFN- $\gamma$ in a paracrine fashion. Human pneumonic lungs and human neutrophils challenged with $L$. pneumophila exhibited increased numbers of IL-17A producing cells. These findings display a novel function of neutrophil-derived IL-17A in antibacterial defense via the induction of IFN- $\gamma$ in a paracrine manner.

\section{INTRODUCTION}

Bacterial pneumonia is a leading cause of mortality, morbidity, and health-care costs worldwide. ${ }^{1}$ The intracellular pathogen Legionella pneumophila causes both nosocomial and community pneumonia termed Legionnaires' disease. ${ }^{2,3}$ In addition to the mouse model of $L$. pneumophila ${ }^{4}$ infection, which mimics Legionnaires' disease in immunocompetent individuals, ${ }^{5,6}$ macrophages from the inbred $\mathrm{A} / \mathrm{J}$ mouse strain are often used in studies as they are permissive for growth of L. pneumophila, ${ }^{6,7}$ whereas macrophages from other inbred mice are not. ${ }^{4}$

Recruitment of neutrophils is a hallmark of bacterial pneumonia. ${ }^{8,9}$ Neutrophil influx into the lung is critical for host defense against $L$. pneumophila, ${ }^{10}$ as the selective depletion of neutrophils leads to impaired bacterial clearance. Th17 (interleukin-17 (IL-17)-producing) T cell subsets are known to be involved in neutrophil migration. ${ }^{11}$ IL-17A and IL-17F are homodimeric cytokines produced by Th17 cells that bind to the same receptors (IL-17RA or IL-17RC). ${ }^{12,13}$ Murine IL-17A is the well characterized $21-\mathrm{kDa}$ glycoprotein with 147 amino acids that shares $63 \%$ amino acid homology with human IL$17 \mathrm{~A}(155 \mathrm{aa}) .{ }^{14}$

In a recent investigation, $I l 17 a / I l 17 f^{-/-}$mice on a Balb/c background were utilized to show that IL-17A/F is important for controlling bacterial burden, inducing neutrophil influx into the lung, and for survival in response to L. pneumophila. ${ }^{15}$ However, to further expand upon this initial study, we used: (1) single and double knockout mice on $\mathrm{C} 57 \mathrm{Bl} / 6$ background and antibody blocking in A/J mice; (2) the role of IL-17A or IL-17F

\footnotetext{
${ }^{1}$ Laboratory of Lung Biology, Department of Pathobiological Sciences and Center for Experimental Infectious Disease Research, School of Veterinary Medicine, Louisiana State University (LSU), Baton Rouge, Louisiana, USA. ${ }^{2}$ Center for Experimental Medicine and Core Research for Evolutional Science and Technology (CREST), Japan Science and Technology Agency, Saitama, Japan and ${ }^{3}$ Division of Pulmonary and Critical Care, Department of Medicine, LSU Health Sciences Center, New Orleans, Louisiana, USA. Correspondence: S Jeyaseelan (jey@lsu.edu)

${ }^{4}$ Present address: Laboratory of Pulmonary Immunotoxicology, Department of Environmental Toxicology, Southern University and A\&M College, Baton Rouge, Louisiana 708013, USA.
} 
individually or together are important for host defense; (3) bone marrow chimeras and adoptive transfer experiments to determine which cell types are responsible for IL-17A and IL-17F production; (4) IL-17 homodimer (IL-17A or IL-17F) or heterodimer (IL-17A/F) to determine which is more effective at inducing host resistance; and (5) IL-17-mediates IFN- $\gamma$ production via paracrine manner to induce host defense.

\section{RESULTS}

Bacterial infection induces production of IL-17A and IL-17F in lungs

To determine whether bacterial infection regulates the production of IL-17A and IL-17F, we performed time course experiments $(24,48$, and $72 \mathrm{~h}$ ) in a well-established mouse model of L. pneumophila infection ( $\left.10^{7} \mathrm{CFUs} / \mathrm{mouse}\right)$. As shown in Supplementary Figure S1A online, IL-17A and IL-17F along with TNF- $\alpha$, IL-12/IL-23 (p40), and IL-23 (p19) were upregulated at the mRNA level and peaked at $6 \mathrm{~h}$ postinfection. As IL-17A and IL-17F can exist as either homo- or heterodimers, ${ }^{16,17}$ we specifically determined the levels of IL-17A, IL-17A/F, and IL-17F in the lungs following infection and found them all to be induced at the protein level in lung tissue, with concentrations peaking at $24 \mathrm{~h}$ postinfection (Supplementary Figure 1B). Using specific ELISA (enzymelinked immunosorbent assay) kits, IL-17A/F expression was detected at much higher levels than either IL-17A or IL-17F at all time points (Supplementary Figure 1B).

Bacterial clearance is dependent on IL-17A, but not IL-17F Although it was recently reported that deletion of both IL-17A and IL-17F genes (Il17a/Il17f ${ }^{-/-}$; double knockout (DKO) mice) results in impaired L. pneumophila clearance in the lungs, the individual roles of IL-17A and IL-17F with regard to $L$. pneumophila infection remained unclear. ${ }^{15}$ To this end, we compared wild-type (WT; C57Bl/6), Ill17a ${ }^{-/-}, I l 17 f^{-/-}$, and Il17a/Il17f $f^{-/}$(DKO) mice. Similar to the findings reported previously, ${ }^{15}$ we found that $I l 17 a / I l 17 f^{-/-}$mice exhibited a higher bacterial burden in the lung and increased dissemination at 24,48 , and $72 \mathrm{~h}$ post infection as compared with their WT counterparts. Intriguingly, compared with $I l 17 f^{-/-}$mice, $I l 17 a^{-/-}$exhibited a higher bacterial burden in the lung at 48 and $72 \mathrm{~h}$ post infection and enhanced bacterial dissemination (liver colony-forming units) at 24,48 , and $72 \mathrm{~h}$ post infection (Figures 1a, b).

Neutrophils are critical to the augmentation of host defense against L. pneumophila. ${ }^{15}$ Attenuated recruitment of total WBCs or neutrophils in airspaces of $1 l 17 a^{-/-}$and $I l 17 a /$ Ill $1 f^{-/-}$mice was observed at both 48 and $72 \mathrm{~h}$ post L. pneumophila infection (Figures 1c, d). Furthermore, neutrophil recruitment to the lung parenchyma, as measured by myeloperoxidase (MPO) activity in lung homogenates of $1117 a^{-/-}$mice, was reduced at 24,48 , and $72 \mathrm{~h}$ following L. pneumophila infection (Figure 1e). Conversely, total WBC and neutrophil counts in airspaces and lung parenchyma of $I l 17 f^{-/-}$mice were not attenuated Ill $17 f^{-/-}$mice (Figures 1c-e), whereas neutrophil recruitment to the lung parenchyma was modestly reduced at 48 and $72 \mathrm{~h}$ after infection in $I l 17 f^{-/-}$mice (Figure 1e).

Consistent with reduced neutrophil accumulation in the lungs, attenuated levels of chemokines (KC/CXCL1, MIP-2/ CXCL2, LIX/CXCL5) were observed in $\mathrm{Il17a}^{-/-}$mice following L. pneumophila infection (Figures 1f-h). As compared with control mice, $I l 17 f^{-/-}$mice exhibited modest or no reduction in either chemokines or cytokines at any of the time points examined (Figures 1f-h). Moreover, lungs obtained from WT (control) mice demonstrated moderate suppurative bronchopneumonia, whereas $I l 17 a^{-/-}$and Il17a/Il17f $f^{-/-}$mice displayed only mild suppurative bronchopneumonia at $72 \mathrm{~h}$ following L. pneumophila infection (Figures 1i, j). Furthermore, Ill $17 f^{-/-}$mouse lungs demonstrated no significant difference in histopathological changes as compared with control mice (Figures 1i, j). These results suggest that IL-17A is an important mediator for host defense and that IL-17A deficiency has a dominant role in Il17a/Il17f $f^{-/-}$mice.

\section{Blocking IL-17A attenuates bacterial clearance in permissive $(\mathrm{A} / \mathrm{J})$ mice}

To validate the findings obtained in an L. pneumophila nonpermissive host (C57Bl/6 strain), we used a permissive host (A/J mice) in which blocking antibodies to IL-17A (1 $\mu$ g per mouse) or IL-17F ( 1 or $10 \mu \mathrm{g}$ per mouse) were administered $1 \mathrm{~h}$ before L. pneumophila challenge. Survival experiments in A/J mice using IL-17A Ab (10 $\mu \mathrm{g}$ per mouse), but not IL-17F Ab, following L. pneumophila infection $\left(10^{8}\right.$ colony-forming units per $50 \mu \mathrm{l}$ per mouse) resulted in reduction in survival (Figure 1k). Intratracheal administration of an IL-17A neutralizing antibody increased bacterial burden in the lungs (Figure 2a) and liver (Figure 2b) as well as decreased recruitment of WBCs and neutrophils to the lungs (Figures 2c, d). Furthermore, blocking IL-17A also reduced chemokine (KC, MIP2) levels (Figures 2e, f). On the other hand, blocking of IL-17F had no significant effect on bacterial clearance, neutrophil influx, or chemokine production following infection (Figures 3a-f).

\section{IL-17A homodimer rescues the impaired bacterial clearance in $1117 \mathrm{a}^{-/-}$mice}

Because IL-17A and IL-17F form not only homodimers, but also a heterodimer (IL-17A/F), ${ }^{11,18}$ we next asked which is effective in rescuing neutrophil-dependent host defense in $1 l 17 a^{-/-}$ mice following L. pneumophila infection. Each homodimer, the heterodimer, or vehicle control (BSA) was administered $1 \mathrm{~h}$ post L. pneumophila infection. Administration of a single dose of IL$17 \mathrm{~A}$ and IL-17A/F, but not IL-17F, augmented bacterial clearance in the lungs (Figure 4a), attenuated bacterial dissemination to liver (Figure $4 \mathbf{b}$ ), and enhanced neutrophil influx (Figures 4c, d) and cytokine/chemokine expression in $I l 17 a^{-1-}$ mice (Figures 4e, f). Intriguingly, exogenous IL-17A is much more potent than IL-17A/F in rescuing host defense function in $I l 17 a^{-/-}$mice following L. pneumophila infection (Figures 4a-f). 

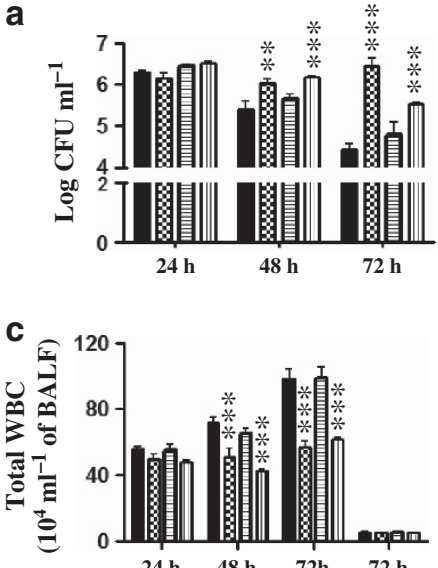

e

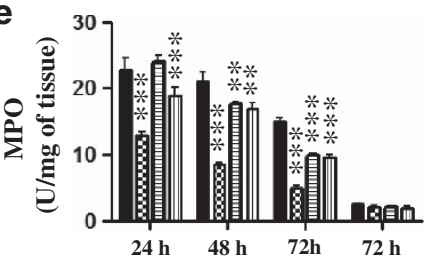

g

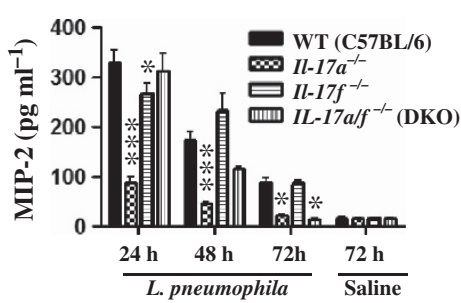

b
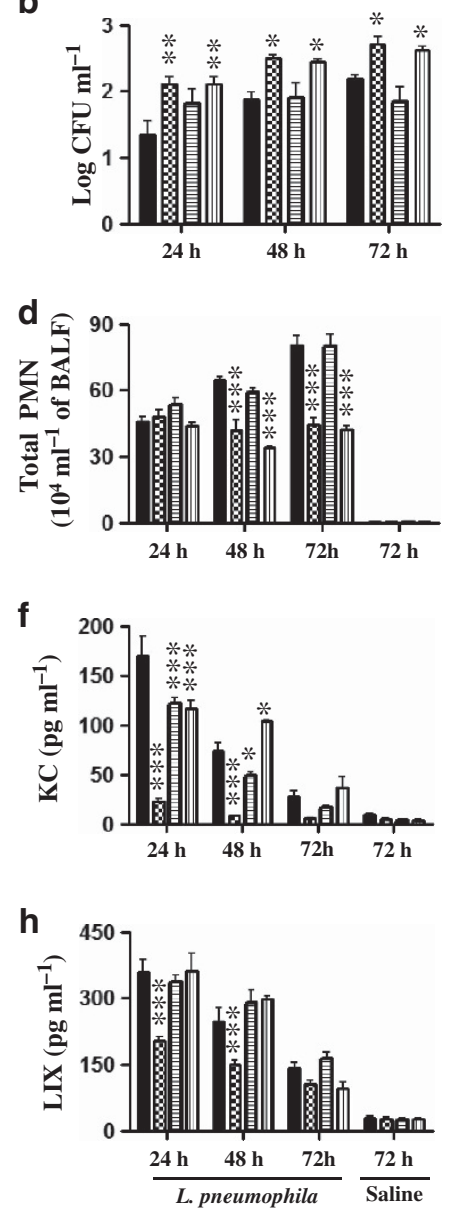

i
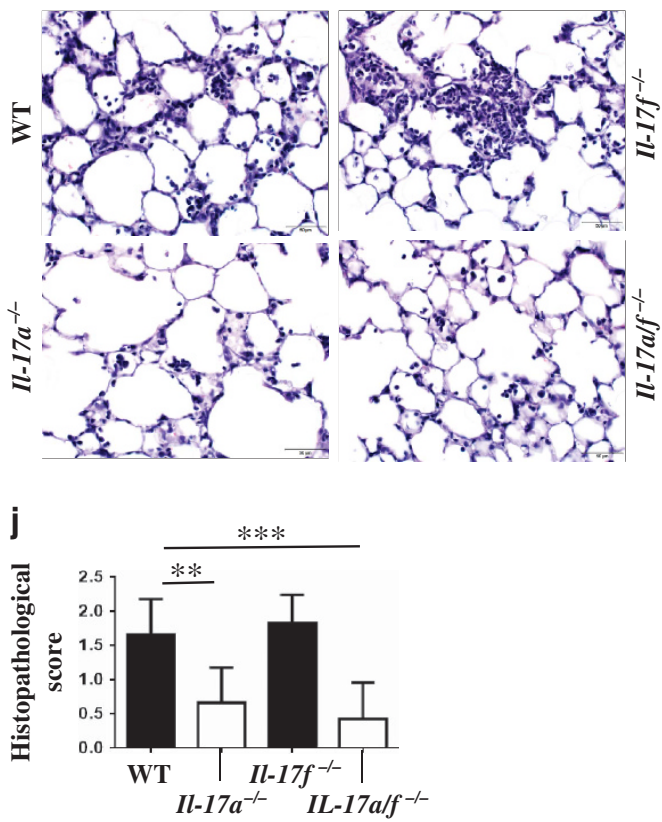

K

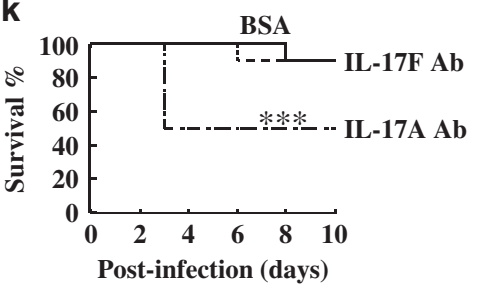

Figure 1 IL-17A mediates bacterial clearance, cellular recruitment, cytokine/chemokine production and survival following $L$. pneumophila infection.

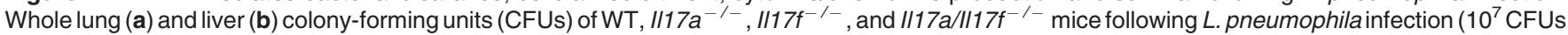
per mouse). Lung or liver homogenates at 24,48 , and $72 \mathrm{~h}$ were used to enumerate the bacterial CFUs. (c-d) BALF was collected at 24,48 , and $72 \mathrm{~h}$ post

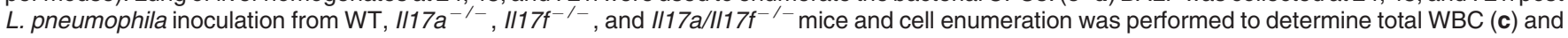
neutrophil (PMN) recruitment (d) to the airspaces. (e) Neutrophil recruitment to the lung parenchyma was determined by myeloperoxidase (MPO) activity in the lungs of $L$. pneumophila-infected mice. ( $\mathbf{f}-\mathbf{h})$. Chemokine levels in BALF at 24,48 , and $72 \mathrm{~h}$ post infection were measured by sandwich ELISA. (i-j) Lung sections were prepared at $72 \mathrm{~h}$ post bacterial infection and were stained with hemotoxylin and eosin. These are representative sections of six mice with identical results at a magnification of $\times 40$ (i). Scores of six histolopathological sections from WT, $/ 117 a^{-1-}, I / 17 f^{-1-}$, and $/ / 17 a / l 17 f^{-/-}$mice were performed in a masked manner (j). For $\mathbf{a}-\mathbf{j}, n=6-9$ mice per group). ${ }^{*} P<0.05 ;{ }^{* *} P<0.01 ;{ }^{* *} P<0.001$; (significance as compared with $L$. pneumophilainfected C57BI/6 mice). (k) IL-17A or IL-17F blocking antibody (10 mg per mouse) administered A/J (control) mice were inoculated intratracheally with $10^{8}$ CFUs of $L$. pneumophila $1 \mathrm{~h}$ post antibody challenge and mortality was monitored up to 10 days $\left({ }^{\star \star \star} P<0.001\right.$ by log rank test; $n=10$ mice per group). BALF, bronchoalveolar lavage fluid; IL, interleukin; WBC, white blood cell; WT, wild type.

PMNs are the major source of IL-17A and IL-17F in lungs A critical event in bacterial pneumonia is the migration of circulating neutrophils to the inflammed tissues. ${ }^{8,9,19}$ To determine the cell types within the infected lung lesions that produce IL-17A and/or IL-17F, we utilized flow cytometry. Our results show increased accumulation of IL-17A + and IL$17 \mathrm{~F}+\mathrm{T}$ cell subsets (CD4, CD8, $\gamma \delta$, and $\mathrm{NK} 1.1+$ cells) compared with neutrophils in infected lungs at $24 \mathrm{~h}$ postinfection, whereas numbers of IL-17A + and IL-17F + neutrophils infiltrating into the lungs were much higher than $\mathrm{T}$ cells at 48 and $72 \mathrm{~h}$ post infection (Figure 5a). Moreover, the numbers of IL-17F + neutrophils were slightly less than IL-17A + neutrophils at 48 and $72 \mathrm{~h}$ post infection (Figure 5a). Furthermore, macrophages isolated from the infected lungs did not produce either IL-17A or IL-17F (data not shown). We then explored whether IL-17A or IL-17F is produced by T-cell subsets and neutrophils on an individual cell basis upon L. pneumophila stimulation and observed that T-cell subsets (CD4, $\gamma \delta$, NK1.1, CD8, neutrophils) and neutrophils produce IL-17A at $24 \mathrm{~h}$ post infection. Remarkably, T-cell subsets produce more IL-17A than neutrophils on an individual cell basis (Figure 5b). However, only $\gamma \delta$ cells produce IL-17F following $L$. pneumophila infection (Figure 5b). Immunocytochemistry was performed on neutrophils isolated from L. pneumophilainfected lung homogenates and bronchoalveolar lavage fluid (BALF) to assess IL-17A expression and ROR- $\gamma \mathrm{t}$, constitutive expression of the transcription factor for IL-17. 
a
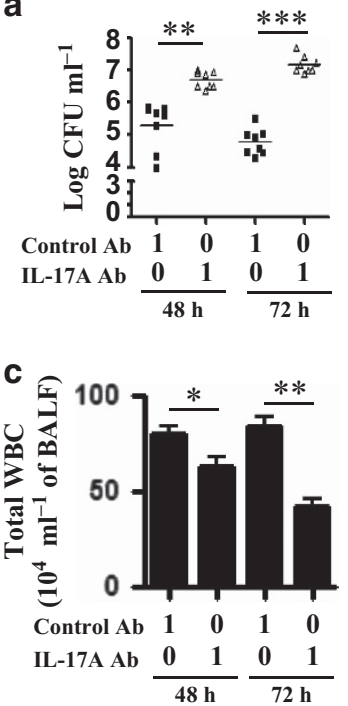

e

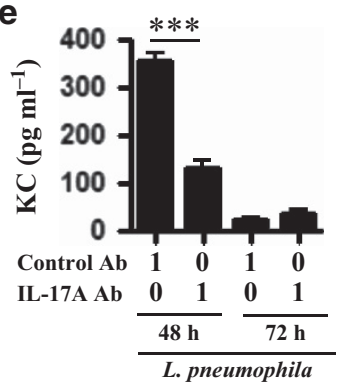

b

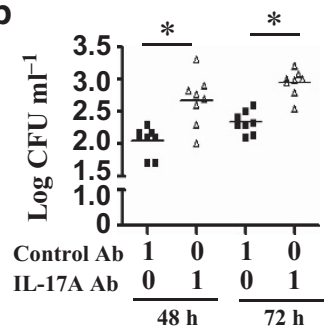

d

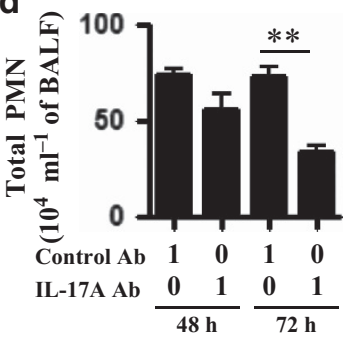

f

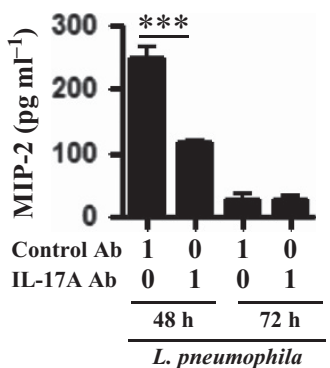

Figure 2 Blockade of IL-17A in A/J mice attenuates the host immune response during pulmonary $L$. pneumophila infection. $(\mathbf{a}, \mathbf{b})$. A/J mice were treated intratracheally with IL-17A blocking antibody ( $1 \mu \mathrm{g}$ per mouse) or control IgG $1 \mathrm{~h}$ before $L$. pneumophila ( $10^{7}$ colony-forming units (CFUs) per mouse) infection. Unlavaged lung (a) and liver (b) were isolated and homogenized and bacterial CFUs enumerated. (c-d). BALF was obtained at 48 and $72 \mathrm{~h}$ post $L$. pneumophila inoculation from blocking Ab or control Ab-treated mice, and cell enumeration was performed to determine total WBC (c) and neutrophil infiltration (d). (e,f). Chemokine levels in BALF were measured by sandwich ELISA following infection with L. pneumophila and Ab blockade. ( $n=6-8$ mice per group). ${ }^{*} P<0.05$; ${ }^{* *} P<0.01 ;{ }^{* \star} P<0.001$; (significance as compared with L. pneumophilainfected C57BI/6 mice). BALF, bronchoalveolar lavage fluid;

ELISA, enzyme-linked immunosorbent assay; IL, interleukin;

WBC, white blood cell.

In neutrophils from uninfected lungs (parenchyma or airspace) minimal ROR- $\gamma \mathrm{t}+$ IL-17A producing neutrophils were detected, whereas neutrophils from infected lungs and BALF exhibited substantial ROR- $\gamma \mathrm{t}+$ IL-17A production at $72 \mathrm{~h}$ postinfection (Figures $\mathbf{5 c}-\mathbf{f}$ ). To further evaluate the ROR- $\gamma \mathrm{t}+\mathrm{IL}-17 \mathrm{~A}$ producing neutrophils, we obtained purified neutrophils from uninfected and infected lungs at $72 \mathrm{~h}$ post infection and using flow cytometry found that neutrophils both from infected lung parenchyma (Supplementary Figure 2A) and airspaces (Supplementary Figure 2B) produce IL-17A. Moreover, we found greater expression of IL-17 receptor A (IL-17RA) than IL-17RC on neutrophils from infected airspaces at $72 \mathrm{~h}$ post infection (Supplementary Figure 2C).

a
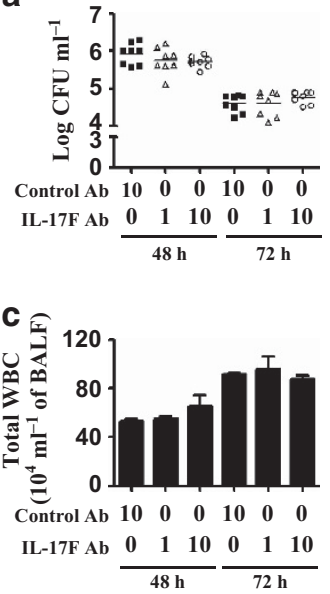

e

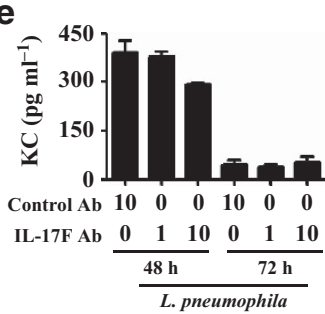

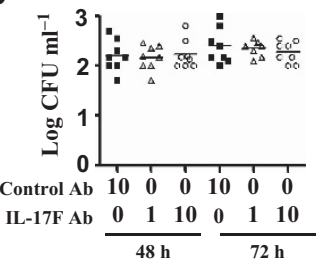

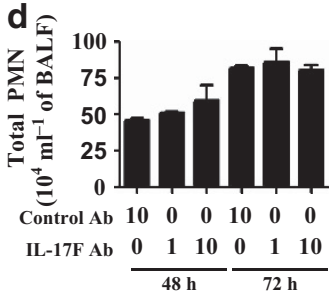

f

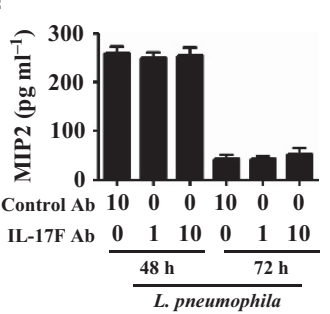

Figure 3 Blockade of IL-17F in A/J mice attenuates the host immune response during pulmonary $L$. pneumophila infection. (a-f). A/J mice were treated intratracheally with IL-17F blocking antibody $(1$ or $10 \mu \mathrm{g}$ per mouse) or control IgG $1 \mathrm{~h}$ after L. pneumophila ( $10^{7}$ colony-forming units (CFUs) per mouse) inoculation. Unlavaged lung (a) and liver (b) were isolated and tissues were homogenized. The lung or liver homogenates were used to enumerate the bacterial CFUs. (c2-d2) BALF was obtained post $L$. pneumophila inoculation from Ab-treated mice, and cell enumeration was performed to determine total WBC (c) and neutrophil recruitment (d). (e,f) Chemokine levels in BALF were measured by sandwich ELISA following antibody blockade and infection with $L$. pneumophila. For experiments a2-f2, $\left(n=6-8\right.$ mice per group). ${ }^{\star} P<0.05$; ${ }^{* *} P<0.01 ;{ }^{* * *} P<0.001$; (significance as compared with L. pneumophilainfected $\mathrm{C} 57 \mathrm{BI} / 6$ mice). BALF, bronchoalveolar lavage fluid; cont-Ab, isotype-matched control antibody; ELISA, enzyme-linked immunosorbent assay; IL, interleukin; WBC, white blood cell.

\section{IL-17 + neutrophils rescue impaired host defense in $1117 a^{-/-}$mice}

We generated bone marrow chimeras to assess the contribution of bone marrow-derived IL-17A versus stromal cell-derived IL$17 \mathrm{~A}$ in bacterial clearance, neutrophil accumulation, and cytokine/chemokine expression. WT (W) $\rightarrow$ WT $(\mathrm{W})$ and WT $(\mathrm{W}) \rightarrow I l 17 a^{-/-}(\mathrm{K})$ mice showed efficient bacterial clearance, whereas $I l 17 a^{-1-}(\mathrm{K}) \rightarrow \mathrm{WT}(\mathrm{W})$ and $I l 17 a^{-1-}$ $(\mathrm{K}) \rightarrow I l 17 a^{-1-}(\mathrm{K})$ mice showed impaired bacterial clearance (Figure 6a). Moreover, in WT $\rightarrow I l 17 a^{-/-}$mice, the rescued host defense effects were blocked by addition of $1 \mu \mathrm{g}$ IL-17A blocking $\mathrm{Ab}$ (data not shown). These studies suggest that IL17A-producing, bone marrow-derived cells predominantly contribute to host resistance. As IL-17+ neutrophils get recruited to the lungs at 48 and $72 \mathrm{~h}$ following L. pneumophila infection, we wanted to determine the functional significance of these cells during $L$. pneumophila infection. To accomplish this, 
we adoptively transferred WT or $1117 a^{-/-}$neutrophils $\left(10^{6}\right.$ cells per mouse) or CD4 T cells ( $10^{6}$ cells /mouse) to recipient $I l 17 a^{-/-}$mice $1 \mathrm{~h}$ post infection. Bacterial clearance, neutrophil accumulation, and cytokines/chemokines including Il-17A were reconstituted in WT neutrophils $\rightarrow$ $I l 17 a^{-/-}$mice but not in $\mathrm{Ill}_{17 a^{-/-}}$neutrophils $\rightarrow \mathrm{Ill} \mathrm{a}^{-/-}$ mice (Figures 6b-1). As compared with WT mice, WT CD4 + T cell infusion into $\mathrm{Ill}_{17 \mathrm{a}^{-/-}}$mice did not result in substantial differences either in bacterial clearance in the lungs or bacterial dissemination to liver (Figures $\mathbf{6 b}, \mathbf{c}$ ), but increases in neutrophil accumulation (Figure $\mathbf{6 d}-\mathbf{f}$ ) and cytokine/ chemokine production (Figures $\mathbf{6 g}-\mathbf{l}$ ) were observed. However, infusion of neutrophils or CD4 $\mathrm{T}$ cells did not induce lung injury as determined by histopathology (data not shown).

\section{Neutrophil depletion increases colony-forming units and reduces IL-17A, IL-17A/F, and IL-17F production in Rag2 $^{-/-}$and Rag2 $^{-/-} /$IIIrg $^{-/-}$mice}

To further explore the role of neutrophils in bacterial burden, and IL-17A, IL-17A/F, and IL-17F production, we depleted neutrophils from Rag2 ${ }^{-/-}$(lack T and B cells; knockout mice-KO) and Rag $2^{-/-} / \mathrm{Il}_{2} \mathrm{rg}^{-/-}$mice (lack T cells, B cells, and NK/NKT cells; Double knockout mice-DKO). Neutrophil depletion in Rag2 ${ }^{-/-}$and $\mathrm{Rag}^{-/-} / \mathrm{Il2} \mathrm{rg}^{-/-}$mice resulted in higher bacterial burden in the lungs (Figure 7a) and reduced IL17A, IL-17A/F, and IL-17F following L. pneumophila infection (Figure 7e-g), confirming that neutrophils are important for bacterial clearance and indeed a major source of IL-17 production. We confirmed neutrophil depletion in the airspaces and lung parenchyma by the Ly6G Ab (Figures $7 \mathbf{b}-\mathbf{d}$ ).

\section{IL-17A is upstream of IFN- $\gamma$ in bacterial clearance}

$I 117 a^{-/-}$mice reconstituted with WT PMNs exhibited enhanced bacterial elimination from lungs and liver (Figures 6b, c) and IFN- $\gamma$ production in the lungs following bacterial challenge (Figure 61). Furthermore, IFN$\gamma$ is known to be critical for control of L. pneumophila multiplication in macrophages and the lungs. ${ }^{20-23}$ In a mouse kidney ischemia-reperfusion injury model, IL-17A has been shown to regulate IFN $-\gamma{ }^{24}$ To determine the relationship between the IL-17A production and IFN- $\gamma$ levels in the lung, exogenous recombinant mouse IFN- $\gamma(50 \mathrm{ng})$ was administered intratracheally to $I l 17 a^{-/-}$mice $1 \mathrm{~h}$ after infection. As compared with controls, IFN- $\gamma$ reduced bacterial burden in the lung (Figure 8a), dissemination (Figure 8b), enhanced PMN recruitment (Figures 8c, d), and chemokine expression (Figures 8e, f). Next, we determined the cellular origin of IFN- $\gamma$ in L. pneumophila-infected lungs and found T cells, but not neutrophils or macrophages, to be the major source of IFN- $\gamma$ in infected lungs (Supplementary Figure 3). On the other hand, administration of exogenous IL-17A ( $1 \mu \mathrm{g}$ per mouse) in Ifn $\gamma^{-/-}$mice $1 \mathrm{~h}$ before L. pneumophila challenge did not decrease the bacterial burden in the lungs or spleen at $72 \mathrm{~h}$ post infection (Supplementary Figure 4). These results suggest that neutrophil-derived IL-17A stimulates IFN- $\gamma$ a

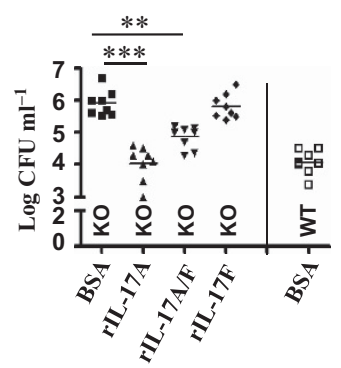

b
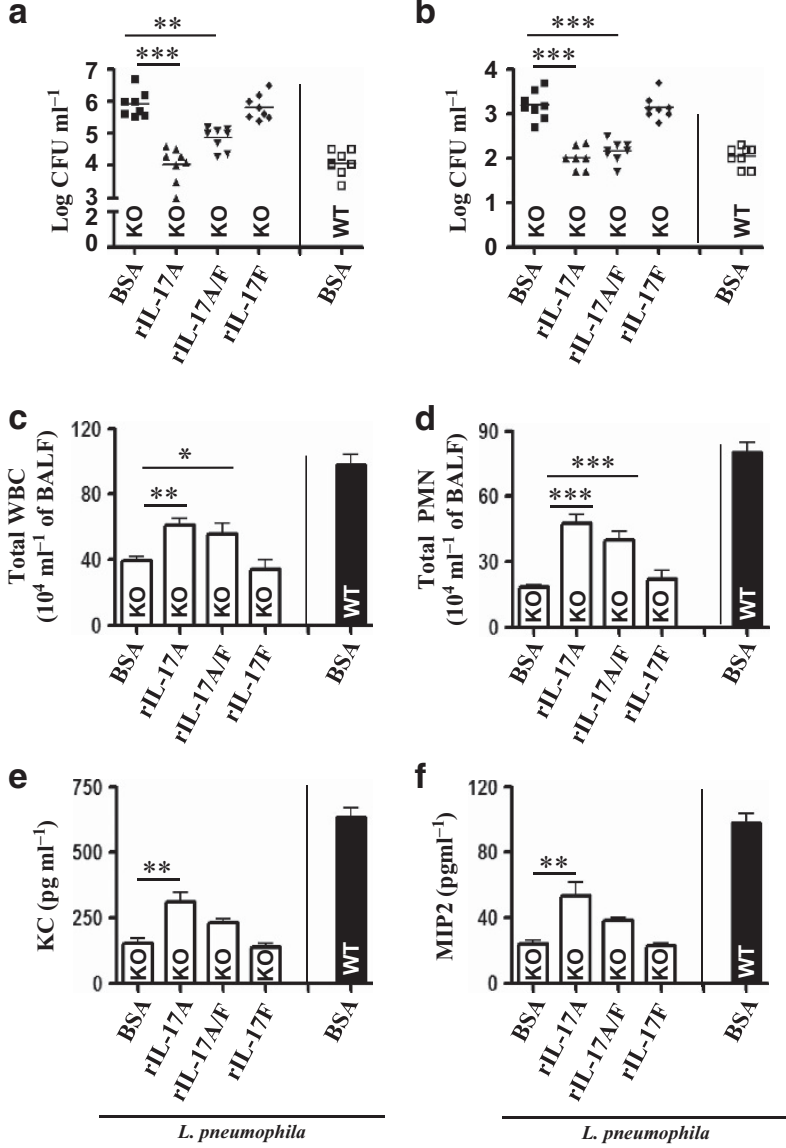

Figure 4 Administration of recombinant IL-17A and IL-17A/F, but not IL$17 \mathrm{~F}$, rescues bacterial clearance, neutrophil recruitment, and cytokine production in the lungs of $11-17 a^{-1-}$ mice following $L$. pneumophila infection. Intratracheal administration of BSA (as control; $1 \mu \mathrm{g}$ per mouse) or $1 \mu \mathrm{g}$ per $50 \mu \mathrm{l}$ per mouse of rm-IL-17A, rmIL-17A/F, or rm-IL-17F to $L$. pneumophila-infected IL-17A ${ }^{-/-}$mice $1 \mathrm{~h}$ post infection. BALF and lung tissues were harvested at $72 \mathrm{~h}$ after $L$. pneumophila infection. Colonyforming units (CFUs) in the lung (a) and liver (b), total leukocytes (WBC) and neutrophils (PMN) in BALF (c,d), cytokine/chemokine levels in BALF and lung homogenates $(\mathbf{e}, \mathbf{f})$ were quantified at $72 \mathrm{~h}$ post infection. *indicates difference between control (BSA) and cytokine-administered mice. $n=6-10$ mice in each group at each time point. ${ }^{*} P<0.05$, ${ }^{* \star} P<0.01,{ }^{\star \star *} P<0.001$. BALF, bronchoalveolar lavage fluid; KO, knockout; IL, interleukin; WBC, white blood cell; WT, wild type.

production via a paracrine manner and IFN- $\gamma$ is critical to control L. pneumophila infection in the lungs.

\section{Human neutrophils produce IL-17A}

To assess whether IL-17A is expressed in lung tissues during human bacterial pneumonia, we performed immunofluorescence staining for IL-17A on lung sections of patients who succumbed to bacterial (Gram-negative) pneumonia and from patients who had died from a non-pulmonary infection. Although control lung sections indicated a small number of lipocalin-2 (Lipo; a neutrophil marker) expressing IL-17A + cells, lung sections from patients who succumbed to bacterial pneumonia showed higher numbers of Lipo $+/$ IL-17A + (double positive) neutrophils (Figures 9a, b). To confirm that IL-17A producing neutrophils are ROR $\gamma$ t-positive in 

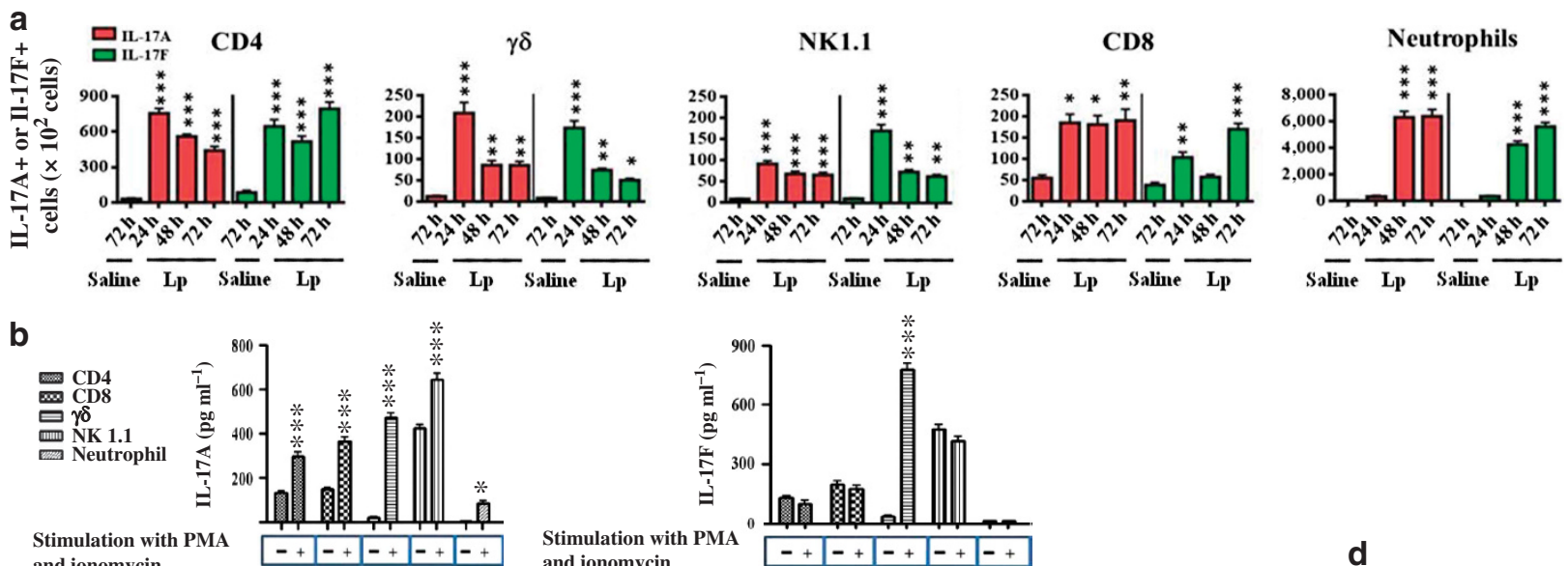
and ionomycin
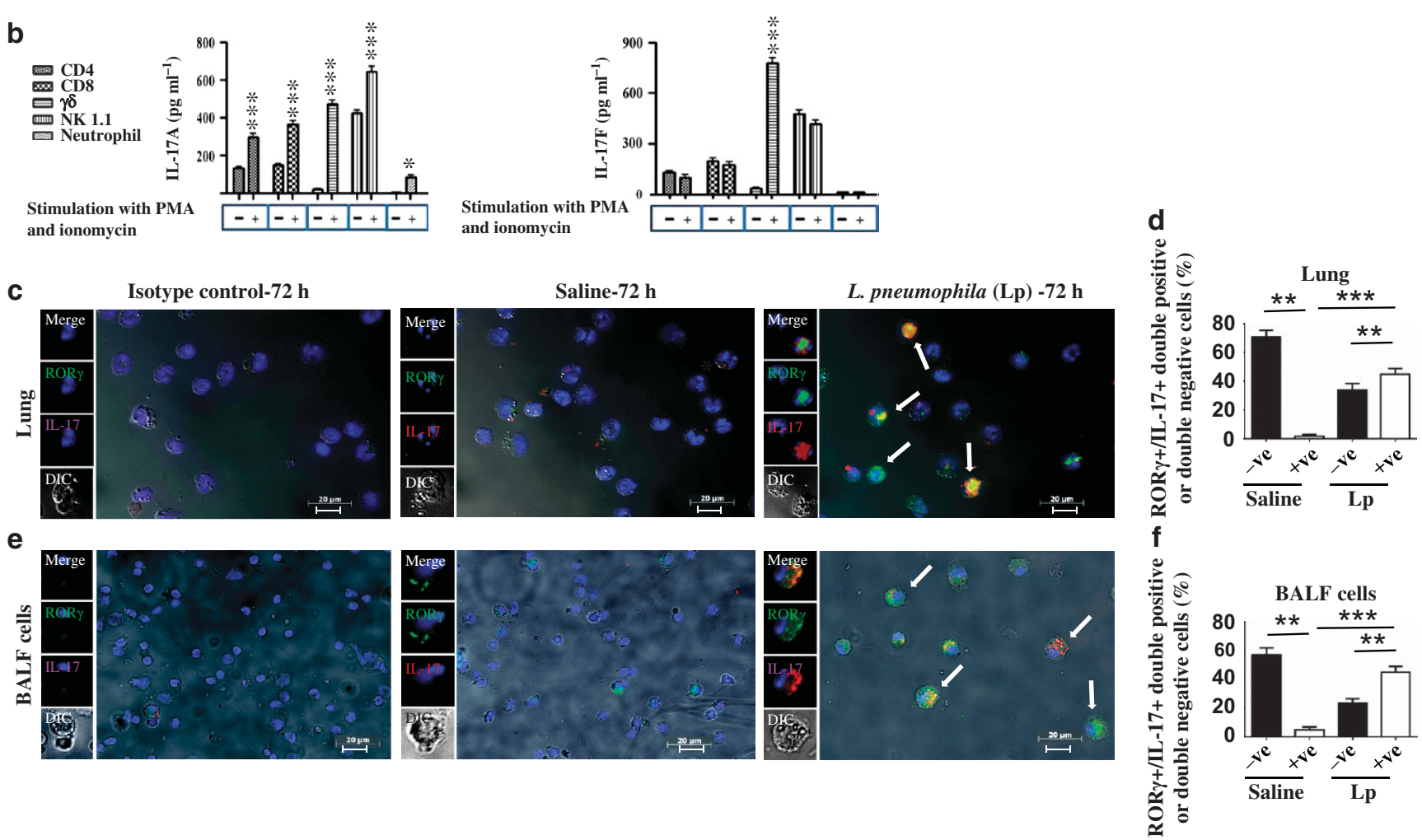

Figure 5 L. pneumophila infection regulates IL-17A and IL-17F production by neutrophils. (a) Flow cytometric analysis was performed on cells obtained from whole-lung digests at 24, 48, or $72 \mathrm{~h}$ post infection with $L$. pneumophila (Lp; $10^{8}$ colony-forming units (CFUs) per mouse) as described in Methods. Total numbers of IL-17A and IL-17F positive cells from each lung following saline challenge or infection. For experiments $\mathbf{a}-\mathbf{b}$, a total of five to eight mice per group were used. ${ }^{*} P<0.05 ;{ }^{* *} P<0.01 ;{ }^{* *} P<0.001$ (compared with $24 \mathrm{~h} L$. pneumophila-infected mice). (b) CD4, $\gamma \delta$, NK1.1, and CD8 cells were purified from the spleen, whereas neutrophils were isolated from digested lungs of $L$. pneumophila-infected mice at $24 \mathrm{~h}$ using magnetic selection (positive selection for $\gamma \delta$ cells; negative selection for CD4, CD8, NK1.1 cells, and neutrophils). Production of IL-17A and IL-17F was determined by ELISA of the culture supernatants following stimulation of $1 \times 10^{4}$ cells with PMA and ionomycin for $5 \mathrm{~h}$. For experiment $\mathbf{b}$, a total of six to eight mice were used to isolate cells. (c-f) Neutrophils express IL-17A and ROR $\gamma$ t following L. pneumophila infection. Neutrophils were purified from the lungs (c,d) or BALF (e,f) and stained with antibodies against IL-17A and ROR $\gamma$ t. Shown is a representative image of six slides with similar results and 500 cells were counted in lung sections or BALF cytospins and plotted. ${ }^{*} P<0.05 ;{ }^{* *} P<0.01 ;{ }^{* \star \star} P<0.001$ (compared with samples with saline challenge). BALF, bronchoalveolar lavage fluid; ELISA, enzyme-linked immunosorbent assay; IL, interleukin.

response to L. pneumophila infection in humans, we purified neutrophils from peripheral blood by negative selection and incubated these cells with L. pneumophila for $3 \mathrm{~h}$ at an MOI (multiplicity of infection) of 1.0. Using immunofluorescence staining, we detected an increase in ROR $\gamma \mathrm{t}+\mathrm{IL}-17 \mathrm{~A}+$ (double positive) neutrophils at $3 \mathrm{~h}$ post infection (Figures 9c, d). Moreover, using flow cytometry, we confirmed that human neutrophils from five healthy donors indeed produce IL-17A, but not IL-17F, following L. pneumophila infection (Figure 9e).

\section{DISCUSSION}

The present study was conducted to determine the role of IL-17A versus IL-17F in lung inflammation and host defense against L. pneumophila pneumonia. Using two completely different approaches, gene-deficient mice on a non-permissive background for L. pneumophila growth and IL-17 neutralization on a permissive background for $L$. pneumophila growth, we established a critical role for the IL-17A homodimer and showed that it is produced primarily by neutrophils during host protection following bacterial challenge. Furthermore, we demonstrated that IL-17A is essential for the production of IFN- $\gamma$, which regulates neutrophil-mediated host defense. Moreover, IL-17F is not only dispensable for the induction of host responses but does not show additive, synergistic, or compensatory effects with IL-17A in these responses. These findings are of interest as both IL-17A and IL-17F are produced by neutrophils and $\mathrm{T}$ cells in the lungs following infection. 

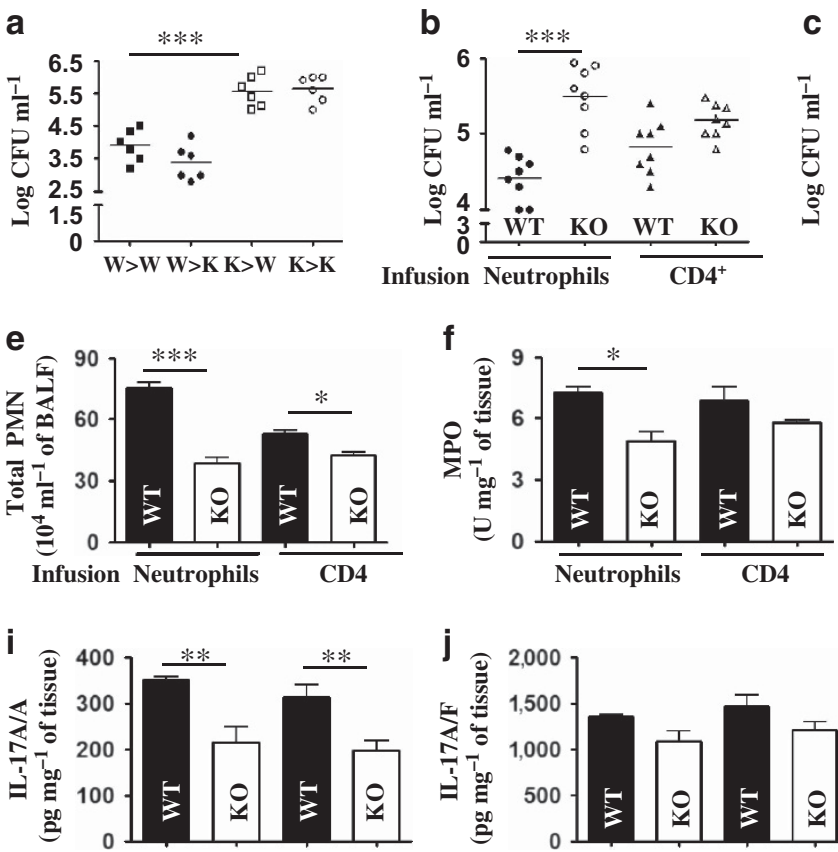

Infusion Neutrophils

$$
\text { L. pneumophila }
$$

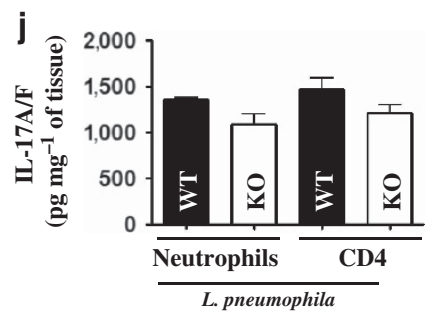

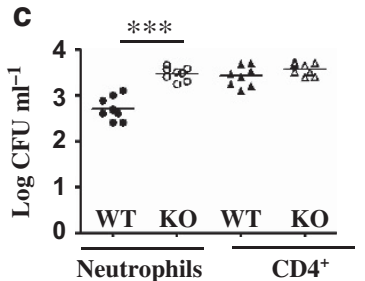
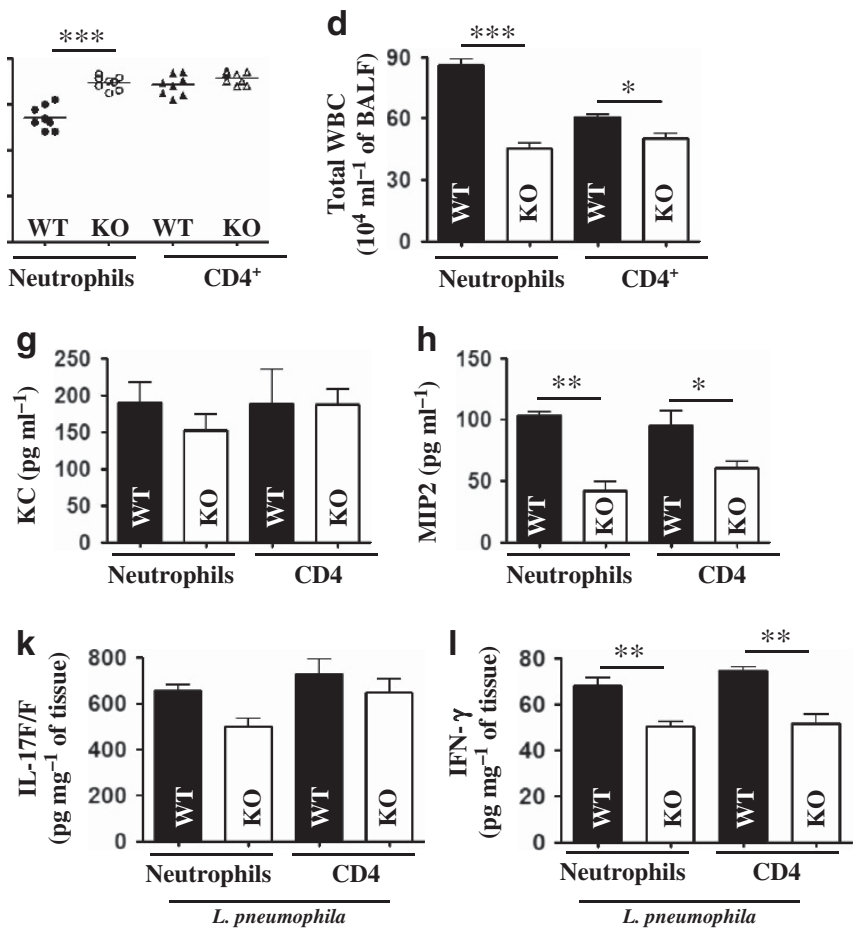

Figure 6 Neutrophil-derived IL-17A rescues bacterial clearance, cellular recruitment, and cytokine expression following L. pneumophila infection. (a) Bone marrow chimeras were generated by lethal irradiation of $/ / 17 a^{-/-}$(knockout (KO) mice; K) and wild-type (WT) mice (W) and reconstitution with bone marrow cells from Kor W mice via tail injection. Bacterial colony-forming units (CFUs) in the lungs were enumerated at $72 \mathrm{~h}$ post $L$. pneumophila infection. ( $n=6$ mice per group). (b-i) Adoptive transfer of neutrophils or CD4 T cells. Bacterial clearance in the lungs (b) and liver (c), leukocyte/neutrophil influx (d-f) and cytokine expression (g-I) in BALF or lung homogenates of IL-17A ${ }^{-/-}$mice $72 \mathrm{~h}$ after L. pneumophila infection followed by adoptive transfer of cells at $1 \mathrm{~h}$ post infection $\left(10^{6}\right.$ cells per mouse; $n=6-9$ mice per group). ${ }^{\star} P<0.05 ;{ }^{\star \star} P<0.01 ;{ }^{\star \star \star} P<0.001$. BALF, bronchoalveolar lavage fluid; IL, interleukin.

a

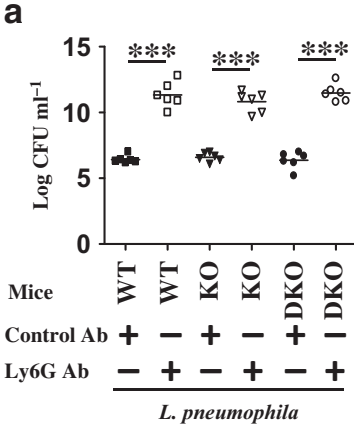

e

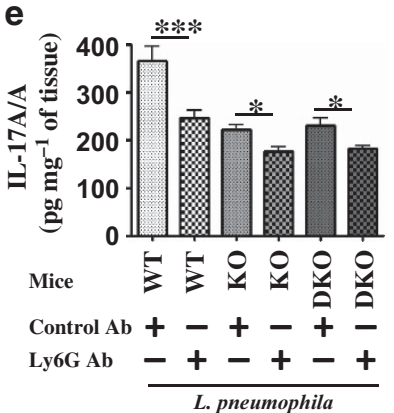

b

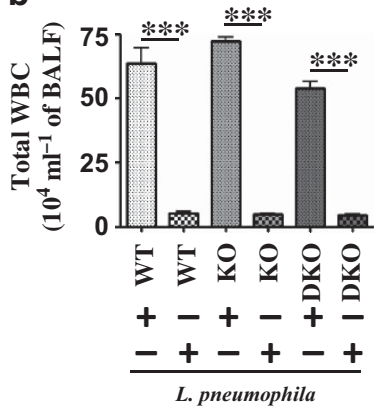

f

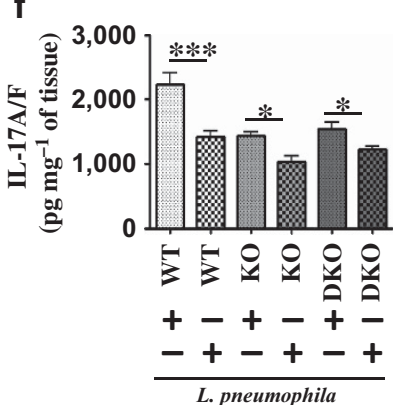

C

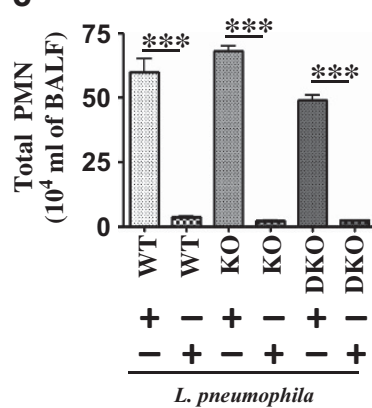

g

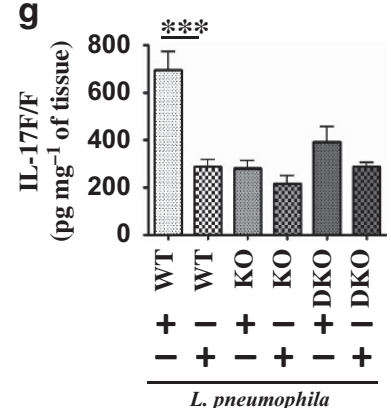

Figure 7 Neutrophil depletion in Rag2 ${ }^{-/-}$and Rag2/IIrg ${ }^{-/-}$mice enhances bacterial burden and reduces IL-17A, IL-17A/F, and IL-17F production following L. pneumophila infection. WT, T-cell- and B-cell-deficient Rag2 $2^{-/-}$(KO) mice or T cell-, B cell-, and NK/NKT-cell-deficient Rag2/II2rg (DKO) mice were pretreated with neutrophil (Ly6G) depleting mAb (IA8) at $12 \mathrm{~h}$ and $2 \mathrm{~h}$ before L. pneumophila infection. Bacterial burden in the lungs, leukocyte/neutrophil numbers and cytokine (IL-17A, IL-17A/F, and IL-17F) levels were determined at $72 \mathrm{~h}$ post infection. For a-g, $n=6-8 \mathrm{mice}$ per group; ${ }^{\star} P<0.05$; ${ }^{* *} P<0.01 ;{ }^{* \star} P<0.001$. BALF, bronchoalveolar lavage fluid; CFU, colony-forming unit; Cont-Ab, isotype-matched control antibody; IL, interleukin; WBC, white blood cell. 
a

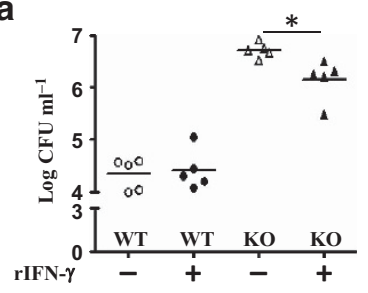

C

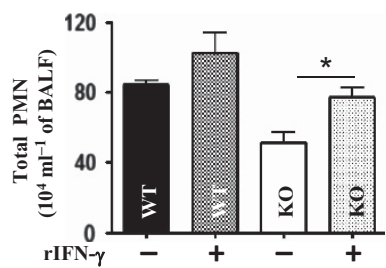

e

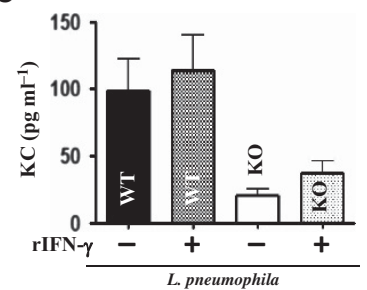

b

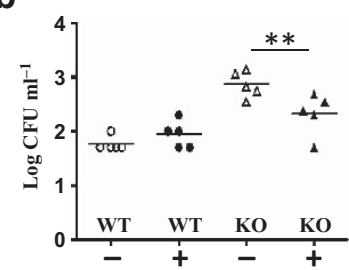

d

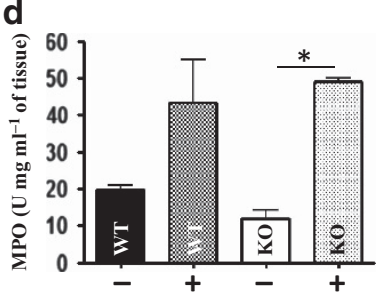

f

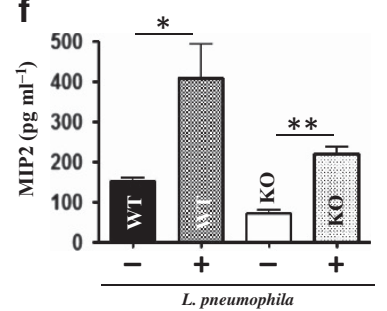

Figure 8 Recombinant IFN- $\gamma$ rescues bacterial clearance, neutrophil recruitment, and chemokine expression following $L$. pneumophila infection in IL-17A KO mice. Mice were intratracheally treated with IFN- $\gamma$ treatment $1 \mathrm{~h}$ post infection with $L$. pneumophila. Bacterial colony-forming units (CFUs) in lungs (a) and spleen (b), leukocyte/neutrophil influx (c,d) and chemokine expression $(\mathbf{e}, \mathbf{f})$ were determined at $72 \mathrm{~h}$ post infection in control (WT) and IL-17A KO mice. $n=5-8$ per group; ${ }^{*} P<0.05$; ${ }^{\star \star} P<0.01 ; * \star * P<0.001$. BALF, bronchoalveolar lavage fluid; ELISA, enzyme-linked immunosorbent assay; IL, interleukin; KO, knockout; WBC, white blood cell; WT, wild type.

IL-17A has been shown to contribute to host responses to a variety of bacterial and fungal agents, such as Klebsiella pneumoniae, Streptococcus pneumoniae, Bordetella pertussis, Yersinia pestis, Mycobacterium tuberculosis, and Candida albicans. ${ }^{25}$ IL-17 is secreted by a variety of cells including T helper type 17 (Th17) cells, CD8, and natural killer (NK) cells, and as a result induces Th17-type host responses characterized by neutrophil recruitment and production of proinflammatory cytokines and chemokines. ${ }^{18,26}$ However, in most of these studies, the role of IL-17F in host defense has not been examined. Previously, a study using IL-17A/F-deficient mice infected with L. pneumophila found reduced neutrophil migration, survival, and production of proinflammatory cytokines (IL-6 and TNF- $\alpha$ ) and chemokines (KC, LIX, and MIP-2); however, it is not possible to determine the individual contributions of IL-17A and IL-17F from this study. In the current investigation, we unequivocally demonstrate the critical role of IL-17A, but not IL-17F in regulating neutrophil-mediated host defense against pulmonary L. pneumophila infection.

Bone marrow-derived cells in the lung produce numerous neutrophil chemoattractants, such as KC and MIP-2, ${ }^{27,28}$ whereas stromal cells, including alveolar epithelial type II cells, produce other neutrophil chemoattractants including LIX. ${ }^{29}$ Our findings are consistent with prior reports of the role of hematopoietic and resident cells during lung inflammation. MyD88 expression in hematopoietic cells is more important for LPS-induced expression of TNF- $\alpha$ and IL-12p40 (ref. 30) than is its expression in stromal cells, despite the fact that both hematopoietic and resident cell-derived MyD88 signaling are essential for LPS-induced neutrophil accumulation. ${ }^{31,32}$ On the other hand, MD-2 signaling in both hematopoietic and resident cells is essential for neutrophil-mediated inflammation and the expression of MIP-2, TNF- $\alpha$, and IL- 6 is mediated by both cell types in the lungs after LPS challenge. ${ }^{33}$ In a similar manner, KC produced by both hematopoietic and stromal cells is important for bacterial clearance and neutrophil recruitment to the lung in response to K. pneumonia infection. ${ }^{9}$ In this study, using bone marrow chimeras and adoptive cell transfers, we specifically identified one hematopoietic cell, the neutrophil, as a key mediator for bacterial clearance in the lungs following L. pneumophila infection.

The hallmark of Th17 cells is expression of the cytokine IL-17A. ${ }^{18,34}$ Interestingly, Th17 cells also express IL-17F, which shares strong homology to IL-17A (refs 11,35) and is expressed from the same chromosomal region. ${ }^{36}$ In previous studies, we demonstrated that $\gamma \delta$ and CD $4+$ T cells are the key producers of IL-17A in the lungs in response to Gram-negative extracellular bacteria, such as E. coli. ${ }^{19}$ Regarding the source of IL-17A and IL-17F, the findings from the current study indicated both $\mathrm{T}$ cells (CD4, CD8, NK 1.1, and $\gamma \delta$ ) and neutrophils produce both IL-17A and IL-17F following L. pneumophila infection, while we did not observe IL-17A or IL-17F production by macrophages following infection (data not shown). Although multiple reports show the production of IL-17A and IL-17F by T-cell subsets during infection, ${ }^{19,37}$ recent studies also demonstrated that IL-17A production by neutrophils in the lung during Aspergillus fumigates and Yersinia pestis infection contributes to host resistance. ${ }^{38}$ In addition, during $Y$. pestis infection, IL-17A produced by neutrophils $(\mathrm{Ly} 6 \mathrm{G}+$ ), and not by $\gamma \delta \mathrm{T}$ or NKT cells, was shown to be important for host protection. ${ }^{39}$

Although prior studies have conclusively demonstrated the effects of mouse IL-17A both in vitro and in vivo non-infectious models, the comparisons between IL-17A and IL-17F were performed using human proteins. The primary conclusion from these studies is that IL-17A is more potent to IL-17A/F in the production of inflammatory mediators from epithelial cells. ${ }^{40}$ In a study using eukaryotic-derived IL-17A and IL-17F homodimers and the heterodimer showed IL-17A was more potent than IL-17A/F, and IL-17A/F more potent than IL-17F in vivo. ${ }^{40}$ Although our observations confirm this in regard to neutrophil recruitment, ${ }^{41}$ our results also show that IL-17A is more potent to IL-17A/F in rescuing bacterial colony-forming units in the lung and liver, neutrophil recruitment and chemokine (KC and MIP-2) production in $\mathrm{Il}-17 \mathrm{a}^{-/-}$mice following L. pneumophila infection. Although the mechanistic differences between human IL-17 and mouse IL-17 signaling 

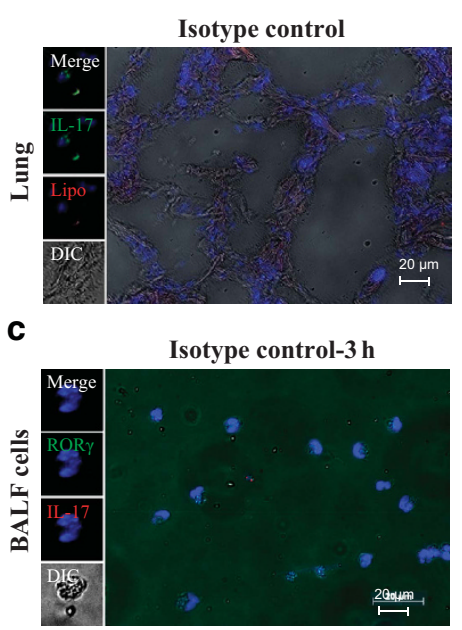

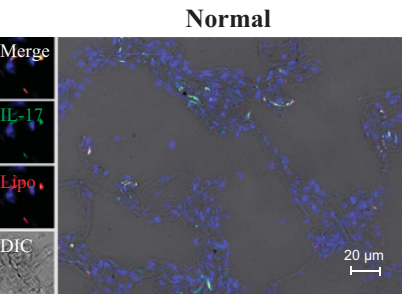

Saline $-\mathbf{3}$ h

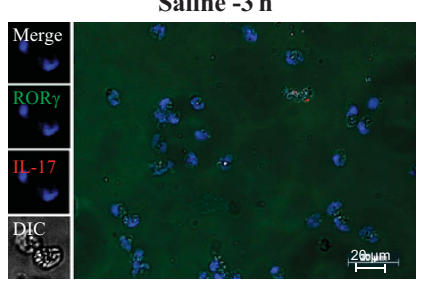

b

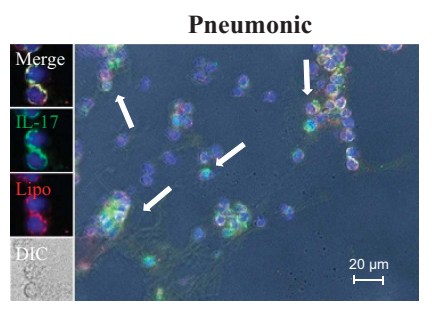

L. pneumophila (Lp) -3 h

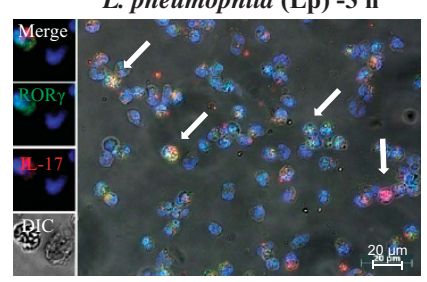

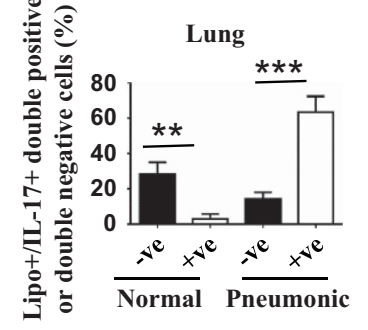

d

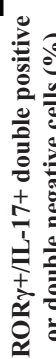

e

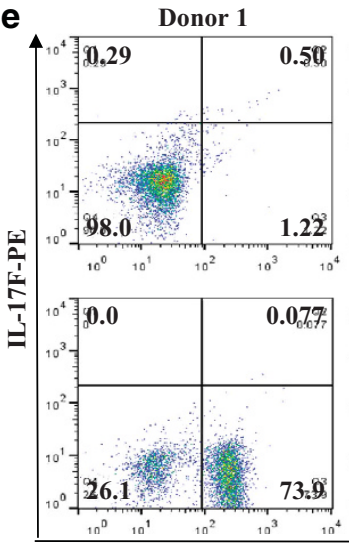

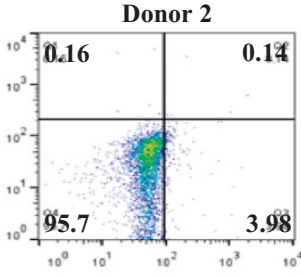
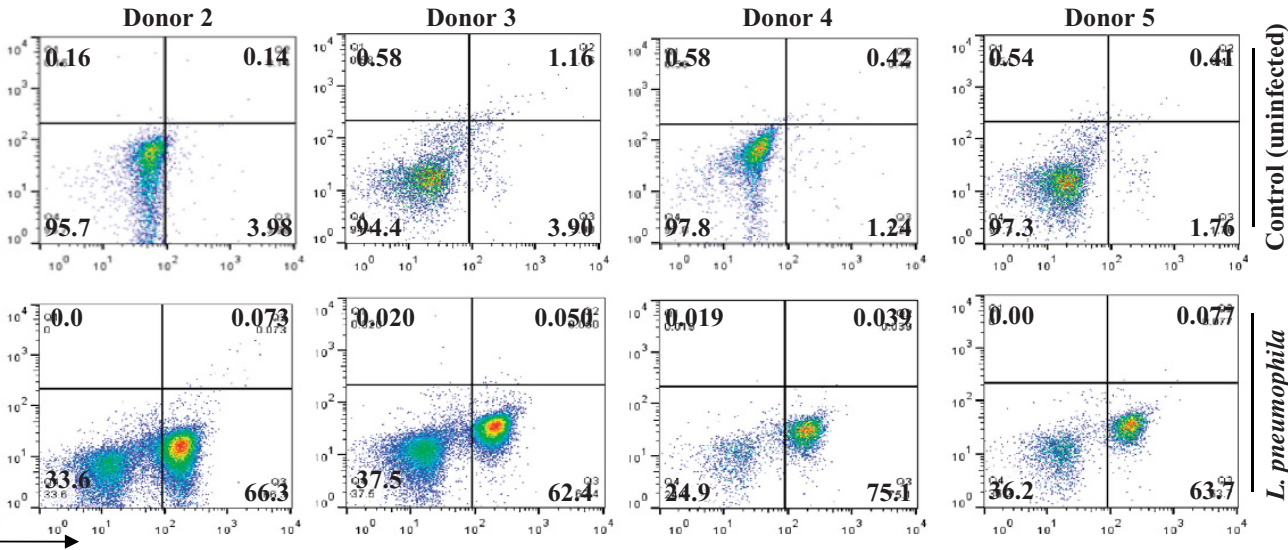

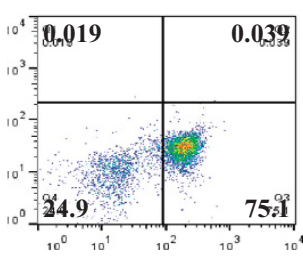

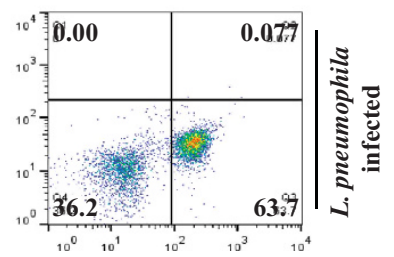

Figure 9 Human neutrophils produce IL-17A following L. pneumophila infection. Bacterial (Gram-negative) pneumonic or non-pneumonic lung sections were stained with lipocalin-2 (lipo; neutrophil-specific) Ab, IL-17A Ab, or control Ab and 500 cells in each section were counted. Shown is a representative section of five lungs from five pneumonic or normal in each group (a) and the mean \pm s.e. of 500 cells from five slides (b). Blood was obtained from healthy volunteers and neutrophils were purified using negative selection with magnetic beads. Neutrophils were stimulated with L. pneumophilaat an MOI of 1.0 . At $3 \mathrm{~h}$ post infection, neutrophils were stained with Abs against ROR $\gamma \mathrm{t}$ and IL-17A and immunofluorescence performed. Shown is a representative image of neutrophils from five healthy individuals (c) and the mean \pm s.e. of 500 cells from five slides (d). Production of IL-17A and IL-17F by neutrophils following L. pneumophila infection. For a-d, ${ }^{\star} P<0.05 ;{ }^{* \star} P<0.01 ;{ }^{* * *} P<0.001$. (e) Human neutrophils were purified and stimulated with $L$. pneumophila at an MOI of 1.0. At $3 \mathrm{~h}$ post infection, neutrophils were stained with IL-17A antibody or IL-17F antibody. The upper panel indicates control or uninfected cells, whereas the lower panel indicates stimulated cells. Dot plot shows IL-17A and/or IL-17F producing cells upon stimulation. BALF, bronchoalveolar lavage fluid; ELISA, enzyme-linked immunosorbent assay; IL, interleukin; MOI, multiplicity of infection.

remain unclear, this may be mediated via different receptor usage. In this regard, human IL-17A and IL-17F induces signaling through two receptors, IL-17RA and IL-17RC, ${ }^{12}$ whereas mouse IL-17A, IL-17A/F, and IL-17F-induced intracellular signaling requires IL-17RA. ${ }^{42}$ As a consequence, receptor binding affinity or avidity may contribute to the differential effect. However, several reports suggest that these proteins may not share the same receptor or may have different functions mediated via distinct signaling cascades. For instance, Starnes et al..$^{43}$ demonstrated that IL-17F can induce TGF- $\beta$ expression in endothelial cells. Furthermore, the same report indicated that IL-17A can enhance angiogenesis in tumor cells, whereas IL-17F can reduce angiogenesis. ${ }^{43}$ From our studies, however, IL-17RA is induced at a greater level in bacteriainfected neutrophils than IL-17RC, suggesting IL-17RA may be the major receptor for IL-17A binding (Supplementary Figure 2C).

Neutrophils are known to have a critical role in host defense during pulmonary $L$. pneumophila infection via immunomodulation, but not via direct bacterial killing. ${ }^{44,45}$ In this context, it has been demonstrated that neutrophils produce IL-12 in the lungs to drive a Th1-type host response leading to IFN- $\gamma$ production. ${ }^{15,46}$ In addition, it is well established that IFN- $\gamma$ is critical for control of L. pneumophila infection in the lungs. ${ }^{21} \mathrm{In}$ this study, we found that neutrophil-derived IL-17 induced IFN- $\gamma$ drives bacterial clearance. However, we did not observe 
IFN- $\gamma$ production by neutrophils following $L$. pneumophila infection (Supplementary Figure 3 ). These results suggest that IL-17A may induce IFN- $\gamma$ through paracrine manner. The observations from this study somewhat differ from those of a prior investigation using $L$. pneumophila in which no differences in IFN- $\gamma$ expression were seen between WT and IL-17A/F-deficient mice because individual contribution of IL-17A or IL-17F was not examined by the previous study. ${ }^{15}$ However, in another study using gene-deficient mice or macrophage depletion, it was shown that IFN- $\gamma$ is important for macrophage-activated protection (M1 phenotype) against $Y$. pestis although the role of neutrophils has not been examined. ${ }^{39}$ The current study indicated that the IL-17AIFN $-\gamma$ signaling axis contributes to innate immune responses associated with L. pneumophila lung infection in paracrine manner (Supplementary Figure 5). In a previous study, it has been shown that infiltrating NKT cells produce IFN- $\gamma$ within a short time $(3 \mathrm{~h})$ of ischemia-reperfusion injury. ${ }^{47}$ In the same investigation, IFN- $\gamma$ was primarily produced by $\mathrm{CD} 1 \mathrm{~d}$-restricted NKT cells and GR-1 ${ }^{+}$neutrophils in kidney following ischemia-reperfusion injury. ${ }^{47}$ Therefore, in the previous study, $\mathrm{CD} 4{ }^{+}$NKT cells provides an explanation for how T cells, the traditional players of the adaptive immune responses, have an essential role in innate immunity. However, future studies are needed to determine the role of this cell type in producing IFN- $\gamma$ in response to IL-17 during L. pneumophila infection.

Hyper IgE syndrome patients have low levels of IL-17 and have recurrent bacterial infections in skin and the lung owing to decreased neutrophil recruitment to tissues. ${ }^{48} \mathrm{~A}$ recent study reported cavitation of Legionella pneumonia in a hyper $\operatorname{IgE}$ syndrome patient, ${ }^{49}$ showing the critical role of IL-17 in human pulmonary Legionella infections. Further, we found IL-17A + neutrophils in bacterial pneumonic lungs and induction of IL-17A in human neutrophils by L. pneumophila infection, validating the results obtained from the preclinical (mouse) model.

Overall, the novel observations in this study unveil a previously unrecognized function of neutrophils to augment host defense via the IL-17A-IFN- $\gamma$ axis and thus, establish a paradigm shift in the way clinicians and researchers think about the role of neutrophils in host defense during bacterial pneumonia followed by sepsis. Moreover, these findings are fundamental to the design of improved treatment and/or prevention strategies to control bacterial pneumonias. In addition, these mechanisms might contribute to inflammation and host defense in response to infections that are caused or complicated by other bacterial pathogens.

\section{METHODS}

Bacterial burden, neutrophil influx, and flow cytometry. Philadelphia 1 strain (ATCC 33152) of L. pneumophila was used. ${ }^{50}$ The bacterium was grown on buffered charcoal-yeast extract agar for 2 days at $37^{\circ} \mathrm{C}$ before use. Experimental L. pneumophila infection and creation of bone marrow chimeras were conducted as described. ${ }^{9,51}$ Bacterial numbers in tissues were enumerated by serial dilutions, whereas neutrophil recruitment in BALF and lung homogenates were measured by total/differential counts and myeloperoxidase assays, respectively. ${ }^{8,9}$ BALF and tissue homogenates were used to measure cytokines using sandwich ELISA kits for KC (R\&D Systems, Minneapolis, MN), MIP-2 (R\&D Systems), LIX (R\&D Systems), IL-17A/A (eBioscience, San Diego, CA), IL-17A/F (eBioscience), IL-17F/F (eBioscience) and IFN- $\gamma$ (R\&D Systems) based on manufacturer's protocols. CD4, CD8, NK1.1, and total T cells were purified from the spleens of L. pneumophila-infected C57Bl/6 mice, whereas neutrophils were obtained from collagenase-digested lungs by negative magnetic selection (StemCell Technologies, Vancouver, British Columbia, Canada). ${ }^{52}$ Neutrophils were purified from the digested lungs of $L$. pneumophila-infected mice using an antibody cocktail (CD5, CD4, CD45R/B220, TER119, F4/80, CD11c, c-KIT) for negative selection, whereas $\gamma \delta$ cells were isolated by positive selection using PE-conjugated anti- $\gamma \delta$ TCR (BD Biosciences, San Jose, CA) as previously described. ${ }^{8}$

Animals, in vivo methods, and tissue isolation. Female $1117 a^{-/-}$

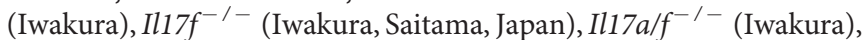
Rag2 $2^{-1-}$ (Taconic, Hudson, NY), and Rag2/Il-2rg ${ }^{-1-}$ (Taconic), and control female mice on a C57BL/6 background (Taconic and Jackson, Bar Habor, ME) were used in compliance with institutional animal use regulations. Neutrophil depletion in mice was induced by intraperitoneal injection of $50 \mu \mathrm{g}$ of anti-Ly6G mAb (clone IA8; $\mathrm{BD}$ Biosciences) or isotype control Ab (clone R35-95; BD Biosciences) at 12 and $2 \mathrm{~h}$ before pathogen challenge as described earlier., ${ }^{8,9,51}$ IL-17A or IL-17F activity was blocked by intraperitoneal administration of $1 \mu \mathrm{g}$ of anti-IL-17A (monoclonal-rat IgG2a, Catalog (Cat) \#MAB421/CF; R\&D Systems), anti-IL-17F (polyclonal, Cat \#AF2057/CF; R\&D Systems), control Ab for anti-IL-17A (rat IgG2a, Cat \#MAB006/CF, R\&D Systems) or control Ab for anti-IL-17F (goat IgG, Cat \#AB-108-C, R\&D Systems) in A/J mice (Jackson). Recombinant IL-17A (Cat \#421-ML/CF, R\&D Systems), IL-17A/F (Cat \#5390-IL/CF, R\&D Systems), IL-17F (Cat \#2057-IL/CF, R\&D Systems), or IFN- $\gamma$ (Cat \#485-MI/CF, R\&D Systems) was administered intratracheally at a concentration of $1 \mu \mathrm{g}$ per mouse, whereas the control mice were administered with phosphate-buffered saline supplemented with BSA (R\&D Systems). For adoptive transfer, naive bone marrow neutrophils or splenic CD $4+\mathrm{T}$ cells $\left(10^{6}\right.$ cells per mouse) were injected via the tail vein. Methods for BALF collection, organ harvest, cell isolation (T cells and neutrophils) and histology were described in earlier publications. ${ }^{8,9}$

Mouse lung preparation for histopathology. Lung tissue was prepared by intratracheal infusion of pressurized, low-melting agarosecontaining formalin. Isolated tissue was placed in $4 \%$ formalin overnight. Five micromolar sections were cut and hematoxylin and eosin staining was performed. Slides were scored for inflammation/ injury by a veterinary pathologist in a masked manner ( $0-4$ scale) using a scoring system described by Matute Bello et al. ${ }^{53}$ Further details regarding this method are provided in the supplement.

Immunohistochemistry. In brief, lung sections were deparaffinized, fixed, and permeabilized with the buffer containing $0.1 \%$ Triton X-100 and then blocked with serum. For mouse lungs, sections were incubated with anti-IL-17 (rabbit polyclonal, Cat \#ab91649, Abcam, Cambridge, MA) or ROR- $\gamma$ (rabbit polyclonal, Cat \#ab78077, Abcam). For human lungs, pneumonic and normal lung sections were incubated with anti-IL-17 (rabbit polyclonal, Cat \#ab136668, Abcam) or Lipocalin-2 (rabbit polyclonal, Cat \#ab41105, Abcam). Rabbit IgG, polyclonal (Cat \#ab27478, Abcam) was used as an isotype control. As a control, lung sections were washed and incubated with Alexa-conjugated secondary antibodies (Invitrogen, Waltham, MA). Lung sections were dehydrated and mounted using Vectashield mounting medium (Vector Laboratories, Burlingame, CA). The slides were viewed using a Zeiss Axioskop 2 Plus microscope.

Immunocytochemistry. The method is the same as described in immunohistochemistry, including antibodies used to demonstrate proteins in lung sections, except we used neutrophils in these experiments. 
Bone marrow transplantation. Donor and recipient $\mathrm{C} 57 \mathrm{Bl} / 6$ mice between 6 and 8 weeks old were used. Recipient mice were gammairradiated with two 525 -rad doses $3 \mathrm{~h}$ apart. Bone marrow cells $\left(8 \times 10^{6}\right.$ per mouse) were injected into the tail vein of the irradiated recipients, and mice were maintained on $0.2 \%$ neomycin sulfate for the first 3 weeks. The reconstituted mice were used for experiments 6 weeks after transplantation. We found that $>78-86 \%$ of blood leukocytes were derived from the donor mice at the time the mice were used for experiments. Irradiated mice that were not transplanted with donor cells died between days 18 and 20 after irradiation (data not shown).

Human ex vivo studies. Peripheral blood was isolated from healthy donors in compliance with institutional regulations and neutrophils were purified using negative magnetic selection (StemCell Technologies, Vancouver, British Columbia, Canada). ${ }^{8}$ These cells were stimulated with $1 \mathrm{MOI}$ of L. pneumophila. At $3 \mathrm{~h}$ post stimulation, cells were fixed and used for immunofluorescence microscopy and flow cytometry.

Statistics. Two-way analysis of variance followed by Bonferroni's post hoc analysis was used for multiple comparisons. Data were analyzed with the Student's $t$-test (between two groups). The results are from two to three independent experiments. Survival curves were compared by the Wilcoxon rank sign test. Unless otherwise stated, all the data are expressed as mean and standard error of the mean (s.e.m.). Statistics were calculated with Prism version 4.0 (GraphPad, La Jolla, CA). Differences were considered significant at ${ }^{\star} P<0.05,{ }^{\star \star} P<0.01$, and ${ }^{* * *} P<0.001$.

SUPPLEMENTARY MATERIAL is linked to the online version of the paper at http://www.nature.com/mi

\section{ACKNOWLEDGMENTS}

This work was supported by Scientist Awards from the Flight Attendant Medical Research Institute (CIA and YCSA to S.J.; YCSA to S.C. and S.B.); and grants from the $\mathrm{NIH}$ (R01 HL-091958, R01 HL-091958S1 via ARRA and R01 Al113720 to S.J. and P30 GM110760 to SB R15 ES023151 to S.B.). We thank the Lung Biology members Mary Leissinger, Taylor Ferguson, Jonte Ellison, Liliang Jin, Ritwij Kulkarni, Pankaj Baral, and K. Jeyagowri for helpful discussions.

\section{DISCLOSURE}

The authors declare no conflict of interest.

c) 2016 Society for Mucosal Immunology

\section{REFERENCES}

1. Mizgerd, J.P. Lung infection—a public health priority. PLoS Med. 3, e76 (2006).

2. Agullo-Ortuno, M.T., Garcia-Mancebo, M.L., Montes-Ares, O. Noguera-Velasco, J.A. Biochemical and immunologic features of an outbreak of Legionnaires disease: comparative study between community-acquired pneumonias. Diagn. Microbiol. Infect. Dis. 56, 7-11 (2006).

3. Almirall, J., Blanquer, J. \& Bello, S. Community-acquired pneumonia among smokers. Arch. Bronconeumol. 50, 250-254 (2014).

4. Losick, V.P., Stephan, K., Smirnova, II, Isberg, R.R. \& Poltorak, A. A hemidominant Naip5 allele in mouse strain MOLF/Ei-derived macrophages restricts Legionella pneumophila intracellular growth. Infect. Immun. 77, 196-204 (2009).

5. Brown, A.S., van Driel, I.R. \& Hartland, E.L. Mouse models of Legionnaires' disease. Curr. Top. Microbiol. Immunol. 376, 271-291 (2013).

6. Brieland, J. et al. Replicative Legionella pneumophila lung infection in intratracheally inoculated $\mathrm{A} / \mathrm{J}$ mice. A murine model of human Legionnaires' disease. Am. J. Pathol. 145, 1537-1546 (1994).

7. Brieland, J.K., Loebenberg, D., Menzel, F. \& Hare, R.S. Efficacy of SCH27899 in an animal model of Legionnaires' disease using immunocompromised A J mice. Antimicrob. Agents Chemother. 44, 1333-1336 (2000).

8. Batra, S., Cai, S., Balamayooran, G. \& Jeyaseelan, S. Intrapulmonary administration of leukotriene $B(4)$ augments neutrophil accumulation and responses in the lung to Klebsiella infection in CXCL1 knockout mice. J. Immunol. 188, 3458-3468 (2012).

9. Cai, S., Batra, S., Lira, S.A., Kolls, J.K. \& Jeyaseelan, S. CXCL1 regulates pulmonary host defense to Klebsiella Infection via CXCL2, CXCL5, NFkappaB, and MAPKs. J. Immunol. 185, 6214-6225 (2010).

10. Tateda, K. et al. Early recruitment of neutrophils determines subsequent T1/T2 host responses in a murine model of Legionella pneumophila pneumonia. J. Immunol. 166, 3355-3361 (2001).

11. Dong, C. Regulation and pro-inflammatory function of interleukin- 17 family cytokines. Immunol. Rev. 226, 80-86 (2008).

12. Chang, S.H. \& Dong, C. Signaling of interleukin-17 family cytokines in immunity and inflammation. Cell Signal. 23, 1069-1075 (2011).

13. Wright, J.F. et al. The human IL-17F/IL-17A heterodimeric cytokine signals through the IL-17RA/IL-17RC receptor complex. J. Immunol. 181, 27992805 (2008).

14. Iwakura, Y., Ishigame, H., Saijo, S. \& Nakae, S. Functional specialization of interleukin-17 family members. Immunity 34, 149-162 (2011).

15. Kimizuka, Y. et al. Roles of interleukin-17 in an experimental Legionella pneumophila pneumonia model. Infect. Immun. 80, 1121-1127 (2012).

16. Zhang, X., Angkasekwinai, P., Dong, C. \& Tang, H. Structure and function of interleukin-17 family cytokines. Protein Cell 2, 26-40 (2011).

17. Ely, L.K., Fischer, S. \& Garcia, K.C. Structural basis of receptor sharing by interleukin 17 cytokines. Nat. Immunol. 10, 1245-1251 (2009).

18. Kolls, J.K. \& Khader, S.A. The role of Th17 cytokines in primary mucosal immunity. Cytokine Growth Factor Rev. 21, 443-448 (2010).

19. Balamayooran, T. et al. RIP2 controls pulmonary host defense to E. coli infection via the regulation of IL-17A. Infect. Immun. 79, 4588-4599 (2011).

20. Schiavoni, G. et al. IFN protects permissive macrophages from Legionella pneumophila infection through an IFN-gamma-independent pathway. J. Immunol. 173, 1266-1275 (2004).

21. Deng, J.C., Tateda, K., Zeng, X. \& Standiford, T.J. Transient transgenic expression of gamma interferon promotes Legionella pneumophila clearance in immunocompetent hosts. Infect. Immun. 69, 6382-6390 (2001).

22. Harding, C.R. et al. The Dot/lcm effector SdhA is necessary for virulence of Legionella pneumophila in Galleria mellonella and AVJ mice. Infect. Immun. 81, 2598-2605 (2013).

23. Plumlee, C.R., Lee, C., Beg, A.A., Decker, T., Shuman, H.A. \& Schindler, C. Interferons direct an effective innate response to Legionella pneumophila infection. J. Biol. Chem. 284, 30058-30066 (2009).

24. Li, L. et al. IL-17 produced by neutrophils regulates IFN-gamma-mediated neutrophil migration in mouse kidney ischemia-reperfusion injury. J. Clin. Invest. 120, 331-342 (2010).

25. Peck, A. \& Mellins, E.D. Precarious balance: Th17 cells in host defense. Infect. Immun. 78, 32-38 (2010).

26. Guglani, L. \& Khader, S.A. Th17 cytokines in mucosal immunity and inflammation. Curr. Opin. HIV AIDS 5, 120-127 (2010).

27. Bozic, C.R. et al. Expression and biologic characterization of the murine chemokine KC. J. Immunol. 154, 6048-6057 (1995).

28. Wolpe, S.D., Sherry, B., Juers, D., Davatelis, G., Yurt, R.W. \& Cerami, A. Identification and characterization of macrophage inflammatory protein 2. Proc. Natl Acad. Sci. USA 86, 612-616 (1989).

29. Jeyaseelan, S. et al. Induction of CXCL5 during inflammation in the rodent lung involves activation of alveolar epithelium. Am. J. Respir. Cell Mol. Biol. 32, 531-539 (2005).

30. Noulin, N. et al. Both hemopoietic and resident cells are required for MyD88-dependent pulmonary inflammatory response to inhaled endotoxin. J. Immunol. 175, 6861-6869 (2005).

31. Skerrett, S.J., Liggitt, H.D., Hajjar, A.M., Ernst, R.K., Miller, S.I. \& Wilson, C.B. Respiratory epithelial cells regulate lung inflammation in response to inhaled endotoxin. Am. J. Physiol. Lung Cell. Mol. Physiol. 287, L143-L152 (2004).

32. Quinton, L.J., Jones, M.R., Robson, B.E., Simms, B.T., Whitsett, J.A. \& Mizgerd, J.P. Alveolar epithelial STAT3, IL-6 family cytokines, and host defense during Escherichia coli pneumonia. Am. J. Respir. Cell. Mol. Biol. 38, 699-706 (2008).

33. Cai, S., Zemans, R.L., Young, S.K., Worthen, G.S. \& Jeyaseelan, S. Myeloid differentiation protein-2-dependent and -independent neutrophil 
accumulation during Escherichia coli pneumonia. Am. J. Respir. Cell. Mol. Biol. 40, 701-709 (2009).

34. Khader, S.A. Th17 cytokines: the good, the bad, and the unknown. Cytokine Growth Factor Rev. 21, 403-404 (2010).

35. Reynolds, J.M., Angkasekwinai, P. \& Dong, C. IL-17 family member cytokines: regulation and function in innate immunity. Cytokine Growth Factor Rev. 21, 413-423 (2010).

36. Yang, X.O. et al. Regulation of inflammatory responses by IL-17F. J. Exp. Med. 205, 1063-1075 (2008).

37. Iwakura, Y., Nakae, S., Saijo, S. \& Ishigame, H. The roles of IL-17A in inflammatory immune responses and host defense against pathogens. Immunol. Rev. 226, 57-79 (2008).

38. Werner, J.L. et al. Neutrophils produce interleukin 17A (IL-17A) in a dectin1- and IL-23-dependent manner during invasive fungal infection. Infect. Immun. 79, 3966-3977 (2011).

39. Lin, J.S., Kummer, L.W., Szaba, F.M. \& Smiley, S.T. IL-17 contributes to cell-mediated defense against pulmonary Yersinia pestis infection. J. Immunol. 186, 1675-1684 (2011).

40. Liang, S.C. et al. An IL-17F/A heterodimer protein is produced by mouse Th17 cells and induces airway neutrophil recruitment. J. Immunol. 179, 7791-7799 (2007).

41. Linden, A., Laan, M. \& Anderson, G.P. Neutrophils, interleukin-17A and lung disease. Eur. Respir. J. 25, 159-172 (2005).

42. Hot, A., Zrioual, S., Toh, M.L., Lenief, V. \& Miossec, P. IL-17A- versus IL17F-induced intracellular signal transduction pathways and modulation by IL-17RA and IL-17RC RNA interference in rheumatoid synoviocytes. Ann. Rheum. Dis. 70, 341-348 (2011).

43. Starnes, T. et al. Cutting edge: IL-17F, a novel cytokine selectively expressed in activated Tcells and monocytes, regulates angiogenesis and endothelial cell cytokine production. J. Immunol. 167, 4137-4140 (2001).
44. Tateda, K. et al. Chemokine-dependent neutrophil recruitment in a murine model of Legionella pneumonia: potential role of neutrophils as immunoregulatory cells. Infect. Immun. 69, 2017-2024 (2001).

45. Horwitz, M.A. \& Silverstein, S.C. Interaction of the Legionnaires' disease bacterium (Legionella pneumophila) with human phagocytes. I. L. pneumophila resists killing by polymorphonuclear leukocytes, antibody, and complement. J. Exp. Med. 153, 386-397 (1981).

46. Friedman, H., Yamamoto, Y. \& Klein, T.W. Legionella pneumophila pathogenesis and immunity. Semin. Pediatr. Infect. Dis. 13, 273-279 (2002).

47. Li, L. et al. NKT cell activation mediates neutrophil IFN-gamma production and renal ischemia-reperfusion injury. J. Immunol. 178, 5899-5911 (2007).

48. Ma, C.S. et al. Deficiency of Th17 cells in hyper lgE syndrome due to mutations in STAT3. J. Exp. Med. 205, 1551-1557 (2008).

49. Di Stefano, F., Verna, N. \& Di Gioacchino, M. Cavitary Legionella pneumonia in a patient with immunodeficiency due to Hyper-lgE syndrome. J. Infect. 54, e121-e123 (2007).

50. Schmeck, B. et al. Legionella pneumophila-induced NF-kappaB- and MAPK-dependent cytokine release by lung epithelial cells. Eur. Respir. J. 29, 25-33 (2007).

51. Cai, S., Batra, S., Wakamatsu, N., Pacher, P. \& Jeyaseelan, S. NLRC4 inflammasome-mediated production of IL-1beta modulates mucosal immunity in the lung against gram-negative bacterial infection. J. Immunol. 188, 5623-5635 (2012).

52. Utting, O. et al. Immune functions in mice lacking Clnk, an SLP-76-related adaptor expressed in a subset of immune cells. Mol. Cell. Biol. 24, 60676075 (2004).

53. Matute-Bello, G. et al. Septic shock and acute lung injury in rabbits with peritonitis: failure of the neutrophil response to localized infection. Am. J. Respir. Crit. Care Med. 163, 234-243 (2001). 